\title{
Le socioécosystème environnement nocturne : un objet de recherche interdisciplinaire
}

\author{
Samuel Challéat ${ }^{*}$ a \\ Géographie et aménagement, Université Toulouse-Jean-Jaurès, UMR 5193 LISST, Toulouse, France
}

Reçu le 6 février 2017. Accepté le 5 décembre 2017

L'environnement nocturne, qui entend caractériser les systèmes humains et non humains fonctionnellement liés à la nuit et à l'obscurité, est un bel objet interdisciplinaire mobilisant des connaissances relevant de l'aménagement, l'astronomie, la biologie et les sciences de la santé. L'auteur nous en propose une synthèse et montre comment la convergence de problématiques hétérogènes permet de constituer un objet «environnement nocturne». Il est intéressant de voir en quoi les différentes critiques dont celui-ci est l'objet sont, à partir du moment où elles entrent en résonance les unes par rapport aux autres, une source d'initiatives locales.

La Rédaction

\begin{abstract}
Résumé - Cet article expose le cheminement par lequel nous constituons l'environnement nocturne en objet de recherche interdisciplinaire. Apparue dans les années 1990 suite à la requalification environnementale de l'éclairage urbain en pollution lumineuse, cette notion floue vise à appréhender les systèmes - humains ou non - fonctionnellement liés à la nuit et à l'obscurité. En nous appuyant sur l'observation des processus de construction, de légitimation et de territorialisation de l'environnement nocturne, nous montrons que cette notion opère une jonction entre différentes acceptions et approches fortement cloisonnées des rôles, fonctions et effets de l'éclairage artificiel nocturne (ALAN, pour artificial light at $n i g h t^{1}$ ). Nous proposons d'aborder son étude suivant le cadre d'analyse des socioécosystèmes qui nécessite la mise en interaction des différentes approches de l'ALAN par les sciences de la société et les sciences du vivant.
\end{abstract}

Mots-clés : environnement / territoire / pollution lumineuse / environnement nocturne / interdisciplinarité

\begin{abstract}
The night-time environment social-ecological system: an interdisciplinary research topic. This paper explains the scientific reasoning that led us to institute the "night environment" as new interdisciplinary research topic from a social-ecological perspective. Sociocultural, ecological and health costs of artificial light at night (ALAN) have been gradually highlighted since the second half of the twentieth century in a range of scientific fields, from astronomy to medicine through ecology and energy. At the same time an environmentalist movement was emerging: the "dark-sky movement" which condemns "light pollution" and carries its demands within local, national or international arenas. In the 1990s, the requalification of urban lighting as light pollution gave rise to the ill-defined notion of night environment. This notion aims to understand the systems, both human and non-human, that are functionally related to the night and darkness. Building on medium and long-term observations of the processes of construction, legitimation and territorialization of the nocturnal environment, we demonstrate that this notion enables to establish a junction between the different strongly compartmentalized meanings and approaches of the roles, functions and effects of ALAN, and more specifically of urban lighting. We propose to approach its study
\end{abstract}

\footnotetext{
*Auteur correspondant : samuel. challeat@univ-tlse2.fr

${ }^{a}$ Samuel Challéat est membre de l'équipe Dynamiques rurales de l'UMR LISST. Il coordonne également le collectif Renoir, https://renoir.hypotheses.org/.

${ }^{1}$ L'acronyme ALAN est utilisé dans cet article par souci de cohérence avec la bibliographie internationale, notamment en sciences expérimentales, sciences de la vie et sciences de la santé.
} 
based on the social-ecological systems framework. This requires creating strong interactions between the different approaches of ALAN: those of the social sciences and those of the experimental, life and health sciences.

Keywords: environment / territory / light pollution / night environment / interdisciplinarity

L'objet de recherche «nuit» connaît un intérêt croissant depuis les années 2000 dans le champ des sciences de la société, et plus particulièrement dans celui de la géographie et celui de l'aménagement des espaces urbains. Ces disciplines appréhendent la nuit urbaine au prisme des modifications spatiotemporelles et économiques engendrées par l'éclairage urbain, outil d'aménagement essentiellement perçu dans ses dimensions positives. Le sillon environnemental est très peu creusé par les sciences de la société : les approches de l'éclairage urbain suivant le référentiel du développement urbain durable sont focalisées sur la question énergétique et la traitent dans ses dimensions techniques et normatives. Pourtant, des coûts socioculturels, écologiques et sanitaires mis en évidence par des champs scientifiques allant de l'astronomie à la médecine en passant par l'écologie, sont portés dans différentes arènes politiques par le Dark-sky movement qui dénonce la «pollution lumineuse». À la fin des années 2000, les expériences territoriales de protection du ciel étoilé se multiplient. Elles élargissent aujourd'hui leur champ d'action à «l'environnement nocturne» dans sa globalité et participent de sa territorialisation: la protection de "l'environnement nocturne» dans ses multiples dimensions socioculturelles, écologiques et sanitaires devient un nouveau principe d'aménagement.

La requalification environnementale de l'éclairage urbain fait émerger la notion floue d'environnement nocturne. Nous nous en saisissons et la constituons en nouvel objet de recherche permettant d'effectuer la jonction entre les travaux des sciences expérimentales, du vivant et de la santé sur les effets et impacts négatifs de l'ALAN et les travaux des sciences de la société - notamment ceux de la géographie de l'environnement - sur la nuit et l'éclairage urbain. L'hypothèse que nous instruisons ici est donc qu'une approche interdisciplinaire forte est la condition sine qua non pour espérer dépasser les approches techniques des problèmes environnementaux afférents à la pollution lumineuse et leurs traitements, souvent fragmentés et disséminés. Dans la perspective d'un tel décloisonnement disciplinaire, le cadre d'analyse socioécosystémique est pertinent. Appréhender l'environnement nocturne comme un socioécosystème permet de mettre en interaction et de faire dialoguer les grands changements actuellement à l'œuvre dans des systèmes jusqu'à présent disjoints. Changements technologiques de l'éclairage tout d'abord (technologie $\mathrm{del}^{2}$, gestion spatiotemporelle différenciée,

\footnotetext{
${ }^{2}$ Pour diode électroluminescente, ou led (light-emitting diode) en anglais.
}

réseaux « intelligents »), changements scientifiques ensuite (les savoirs sur les effets négatifs de l'ALAN et leur diffusion hors des sphères expertes) et enfin, à l'interface, changements territoriaux (nouveaux outils et dispositifs de territorialisation de l'environnement nocturne).

Pour constituer l'environnement nocturne en objet de recherche et en montrer la dimension socioécosystémique et les capacités interdisciplinaires, nous utilisons diverses sources. Tout d'abord, nous mobilisons dans le temps long ${ }^{3}$ la littérature scientifique afin d'en extraire les grandes approches disciplinaires de la nuit, de l'éclairage urbain et des effets négatifs de l'ALAN. Par ailleurs, notre analyse mobilise l'observation - souvent participante - de moyen terme des principales arènes locales, nationales et internationales de discussion de la pollution lumineuse : milieu associatif de l'astronomie amateur, associations environnementales, associations de professionnels de l'éclairage et réseaux scientifiques. Parallèlement, le suivi des évolutions législatives, réglementaires et normatives nous informe sur la constitution de ce problème environnemental en problème public. Enfin, l'immersion dans plusieurs territoires d'action en matière de lutte contre la pollution lumineuse nous permet l'observation directe et in situ des différents processus de territorialisation de l'environnement nocturne ainsi que le suivi des débats que ces derniers génèrent.

Nous présentons dans la première partie les grandes approches de la nuit et de l'éclairage urbain par les champs de l'aménagement, de l'urbanisme et de l'énergie. La deuxième partie souligne la construction dans le temps long, par les sciences expérimentales, du vivant et de la santé, de l'éclairage artificiel nocturne comme pollution. Enfin, la troisième partie montre comment la notion d'environnement nocturne permet de mieux saisir les contours et la portée des actions publiques et collectives en matière de territorialisation de la lutte contre la pollution lumineuse et constitue un champ fertile pour une approche socioécosystémique.

\footnotetext{
${ }^{3}$ Cet article prolonge un travail de doctorat de géographie initié en 2006 et soutenu en 2010 à l'Université de Bourgogne (Challéat, 2010). Il l'enrichit d'une analyse des évolutions législatives et des expériences récentes de territorialisation de l'environnement nocturne menées par plusieurs territoires, qu'ils soient urbains, périurbains ou ruraux.
} 


\section{Les nuits urbaines et éclairées des sciences du territoire}

\section{Les approches essentiellement fonctionnalistes d'un éclairage public héritier de «l'éclairer plus »}

L'éclairage urbain est un outil d'aménagement. Son développement a indéniablement augmenté le confort et la qualité de vie dans les territoires. Pour repousser la tombée de la nuit, les sociétés déploient leurs techniques, dont l'éclairage artificiel, et plus particulièrement celui des espaces extérieurs qui reconfigure la ville et entraîne son extension temporelle, spatiale et économique (Deleuil, 2009). Plusieurs travaux se sont penchés sur les conséquences de l'éclairage urbain en termes de paysages et de reconfigurations sociospatiales de la ville nocturne dans le temps long (émergence du noctambulisme, «nocturnalisation» des sociétés urbaines occidentales, etc.), ainsi que sur le renouvellement des politiques urbaines nocturnes (Nye, 2015). À partir des années 1980, en Grande-Bretagne notamment, de nombreux travaux construisent le champ de la night-time economy (Lovatt et O'Connor, 1995). Ils portent la focale sur les questions de sécurité (suivant le paradigme de la prévention situationnelle et la théorie de l'espace défendable) et les politiques de prévention (santé, hygiène et salubrité publiques, criminalité) (Painter, 1996 ; Brands et al., 2015), souvent à partir d'approches par les frontières, les groupes sociaux ou les outils des ethnic studies entre géographie, sociologie, anthropologie et science politique (Hollands, 2002; Hobbs, 2003; Hadfield et al., 2009; Hadfield, 2014). De son côté, la recherche française aborde la nuit essentiellement sous l'angle des politiques urbaines, plus particulièrement via la fabrique technique et sociopolitique de l'éclairage public (Deleuil, 2009; Deleuil et Toussaint, 2000 ; Mosser et Devars, 2000), la question des temps, du chrono-urbanisme et des mobilités (Gwiazdzinski, 2003; Mallet, 2009; Comelli, 2015), ou encore la gestion des conflits d'usages des espaces-temps nocturnes (Beauparlant et al., 2006; Pieroni, 2014). Schématiquement, la géographie, l'urbanisme et l'aménagement abordent la nuit de façon très partielle : en contexte urbain éclairé, et en soulignant les bénéfices de l'éclairage artificiel compris comme outil positif d'aménagement en termes de commodités, de sécurisation des biens et des personnes, d'esthétique urbaine ou de marketing territorial.

La place de l'obscurité dans les politiques d'éclairage urbain n'est que très récemment discutée, comme en témoignent les travaux de Tim Edensor (2015), de Josiane Meier et al., (2015), de Sylvain Bertin et Sylvain Paquette, (2015), ou encore quelques numéros thématiques de revues sur la nuit urbaine qui intègrent cette question (Van et al., 2015; Gwiazdzinski, 2015). Plus encore, l'approche environnementale de l'éclairage urbain au-delà des aspects énergétiques, parce qu'elle nécessite d'opérer un pas de côté par rapport au mainstream de la pensée urbanistique et aménagiste, est quasiment inexistante dans ces champs de recherche. C'est en 2010 qu'une première thèse de doctorat en géographie et aménagement est publiée en France sur la problématique de la pollution lumineuse (Challéat, 2010). Par la suite, d'autres travaux empruntent cette voie (Challéat, 2011; Challéat et Lapostolle, 2014; Challéat et al., 2015a; 2016; Bourgeois, 2016).

\section{La question énergétique et l'efficience en éclairage public : la nécessité du «consommer moins »}

Au niveau mondial et selon l'Unep (United Nations Environment Programm), l'éclairage artificiel compte pour plus de $15 \%$ de la consommation électrique et environ $5 \%$ des émissions mondiales de gaz à effet de serre $^{4}$. À la même échelle et avec 4 milliards de lampes actuellement en fonction ${ }^{5}$, l'éclairage public est un poste non négligeable de cette consommation. L'Union européenne connaît actuellement une phase très dynamique d'augmentation globale de ses émissions de lumière artificielle. Elle compte sur son territoire 90 millions de luminaires urbains; $75 \%$ d'entre eux ont plus de 25 ans $^{6}$ et l'on estimait encore à 20 millions, au début des années 2010, le nombre de points lumineux extérieurs au mercure haute pression ${ }^{7}$. Pour les collectivités françaises, l'éclairage public représente en moyenne $16 \%$ de la consommation énergétique totale des communes, $17 \%$ de leurs dépenses d'énergie et $41 \%$ de leur facture électrique ${ }^{8}$. La réponse européenne passe par la directive sur l'écoconception ${ }^{9}$ et par la révision récente de la norme d'éclairage EN $13201^{10}$, qui cherche désormais une plus grande efficience énergétique, et donc le chemin vers des économies budgétaires pour les

\footnotetext{
${ }^{4}$ Voir à ce sujet, https://united4efficiency.org/launch-globallighting-challenge/.

5 afe-eclairage.fr, 2017. L'éclairage en chiffres, www.afeeclairage.fr/afe/l-eclairage-en-chiffres-26.html.

${ }^{6}$ Ibid.

${ }^{7}$ Memorandum of understanding for the implementation of a European concerted research action designated as COST Action ES1204: Loss of the Night Network (Lonne) : https://eservices.cost.eu/files/domain_files/ESSEM/Action_ES1204/ $\mathrm{mou} / \mathrm{ES} 1204-\mathrm{e} . \mathrm{pdf}$.

${ }^{8}$ Afe-eclairage.fr, 2017, op. cit.

${ }^{9}$ Directive 2009/125/CE du Parlement européen et du Conseil du 21 octobre 2009 établissant un cadre pour la fixation d'exigences en matière d'écoconception applicables aux produits liés à l'énergie, Journal officiel de l'Union européenne, 31 octobre 2009, L 285/10.

${ }^{10}$ Voir https://standards.cen.eu/dyn/www/f?p=204:32:0:::: F S P ORG ID, F P LANG ID: 6150,34 \& c s =

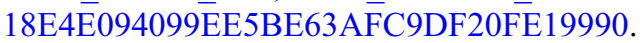


territoires. Plusieurs études soulignent néanmoins le risque d'effet rebond ${ }^{11}$ : au Royaume-Uni, par exemple, l'efficacité lumineuse ${ }^{12}$ a doublé au cours des cinquante dernières années quand, dans le même temps, la consommation d'électricité par habitant pour l'éclairage a quadruplé (Fouquet et Pearson, 2006).

Les champs de l'urbanisme et de l'aménagement se penchent sur ces aspects normatifs et techniques suivant le paradigme du développement urbain durable. Ces travaux se focalisent sur l'optimisation des consommations, la performance des équipements, les caractéristiques (puissance, durée de vie, température de couleur, etc.) et l'efficacité des sources lumineuses ou l'étude des nouveaux modes de gestion de la consommation et des réseaux (Kostic et Djokic, 2009; Radulovic et al., 2011). La technologie del et les réseaux intelligents occupent ici le devant de la scène, aux côtés des systèmes de télésurveillance et de télégestion. Ce faisant, la dimension environnementale de l'éclairage urbain est souvent réduite à sa portion congrue : l'efficience énergétique $^{13}$. Et si les fabricants de luminaires ont, ces dernières années, fortement travaillé le design des lanternes d'éclairage public afin de mieux contenir le flux lumineux à l'intérieur de la « surface utile», ils restent guidés dans cette entreprise par des préoccupations d'économies énergétiques et budgétaires bien plus que par des considérations environnementales. Ainsi, à la fin des années 2000, les trois principaux outils incitatifs proposés aux collectivités pour cheminer vers «un éclairage urbain durable»-certificat d'économies d'énergie, contrat de performance énergétique et circulaire «État exemplaire» du 3 décembre 2008 -ne considèrent que la dimension énergétique de l'éclairage urbain. Il n'en reste pas moins que ces évolutions tracent le sentier d'un nouveau référentiel d'action : «l'éclairer juste».

\section{Le renouvellement de la fabrique de l'éclairage urbain : «l'éclairer juste»}

Au début des années 2010, les technologies flexibles d'éclairage deviennent accessibles pour les collectivités territoriales. Elles offrent de nouveaux possibles à la

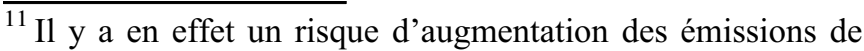
lumière artificielle et de la consommation énergétique suite à l'introduction sur le marché de technologies d'éclairage plus efficaces.

${ }^{12}$ L'efficacité lumineuse d'une source est le rapport entre le flux lumineux émis par cette source lumineuse et la puissance absorbée par la source.

${ }^{13}$ Dans un système physique, l'efficience énergétique est atteinte lorsque la consommation est minimisée pour un service donné. Dans le cas de l'éclairage artificiel, tout éclairage en dehors de la «surface utile» (le service à rendre) va à l'encontre de la minimisation de la consommation, faisant ainsi diminuer l'efficience énergétique de l'installation et ce, même si l'efficacité lumineuse de la source est optimale.
}

fabrique de l'éclairage urbain: sources permettant la gradation de l'intensité lumineuse, systèmes de gestion différenciée - dans l'espace comme dans le temps - du parc d'éclairage public d'un territoire, ou encore technologie d'adaptation des flux à la demande ou suivant la présence ou l'absence d'usagers. Parallèlement, les difficultés budgétaires des collectivités et l'injonction à la transition énergétique rebattent les cartes de la fabrique sociopolitique de l'éclairage urbain et certaines villes entament la reconversion de leur éclairage public, le réduisant ou le coupant durant certaines plages horaires. Ce nouveau référentiel d'action, «l'éclairer juste ${ }^{14} »$, appelle la prise en considération des problèmes des publics par le prisme des territorialités nocturnes (Raffestin, 1988), entendues comme les pratiques et les usages quotidiens dans et de la nuit (Lapostolle et al., 2015). C'est à partir de leur connaissance qu'un éclairage urbain porteur des multiples dimensions économiques, sociales et environnementales du projet urbain peut être défini.

Le passage de la lumière planifiée aux territorialités nocturnes illustre le changement induit par «l'éclairer juste $»$ : combiner controverse sociotechnique (Challéat et Lapostolle, 2014) et territorialités apparaît comme une méthode de politique pragmatique qui laisse au bricolage toute sa place pour sortir de la confiscation cognitive (Lapostolle et al., 2016) de la fabrique de l'espace et de son éclairage ${ }^{15}$. Cette approche bottom-up pluralise la fabrique de l'éclairage urbain. Elle donne une voix, dans la décision, aux habitants (auxquels on demande, par des enquêtes de terrain, des observations participantes, des questionnaires, des réunions publiques plus ou moins formelles, de dire et de décrire leurs besoins et leurs usages de l'espace durant la nuit) et intègre de plus en plus les problématiques environnementales, au-delà des seuls aspects énergétiques.

L'expérimentation mise en œuvre au sein de l'agglomération grenobloise, dans la commune de Crolles (Isère, 8371 habitants en 2014), en atteste. Sur une période de dix mois, de février à novembre 2015, les

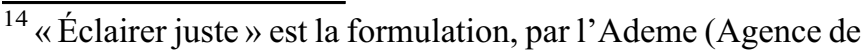
l'environnement et de la maîtrise de l'énergie), des volontés d'évolutions économiques, sociales et aujourd'hui environnementales du design de l'éclairage urbain. Voir www.ademe.fr/ eclairer-juste.

${ }^{15} \mathrm{La}$ «confiscation cognitive» se rencontre dans les démarches expertes, techniques et standardisées d'un urbanisme de plan qui, par la pratique du zonage fonctionnaliste, cible davantage les territoires que les populations et leurs diversités d'usages. Sortir de la confiscation cognitive de la fabrique de l'espace, c'est porter attention, accorder une valeur et donner une voix à l'ensemble des usagers - humains et non humains -, par exemple dans les projets d'éclairage (Challéat et Lapostolle, 2017).
} 
habitants de la ville ont été entendus lors de réunions publiques, au cours desquelles ils ont pu dire leurs craintes, leurs attentes face à l'extinction de l'éclairage public durant certaines tranches horaires (de $1 \mathrm{~h}$ à $5 \mathrm{~h}$ ). Des questionnaires sur l'expérimentation et des promenades nocturnes ont été réalisés pour connaître les besoins et les habitudes de la population. Les changements de comportements liés à la sécurisation des déplacements nocturnes à pied et à vélo, les conseils en matière d'éclairage domestique sont aussi abordés lors des réunions publiques. Cette démarche a permis aux habitants de dépasser certaines représentations anxiogènes liées à l'obscurité, autant qu'elle a diffusé des savoirs scientifiques sur la santé et l'environnement ${ }^{16}$. Après cette période d'expérimentation et de concertation, la plage d'extinction a évolué : l'éclairage public est désormais éteint de $0 \mathrm{~h} 30$ à $4 \mathrm{~h} 30$ en semaine et de $2 \mathrm{~h}$ à $6 \mathrm{~h}$ du matin dans la nuit du samedi au dimanche ${ }^{17}$. Ce nouveau référentiel de «l'éclairer juste» participe de la territorialisation de préoccupations émergentes construites dans le temps long: la «qualité de l'obscurité» et la protection de «l'environnement nocturne » face à l'ALAN.

\section{La qualité de l'obscurité face à l'ALAN, préoccupation des astronomes et des sciences du vivant}

\section{L'ALAN contre le ciel : la dégradation d'un outil de travail et d'un objet de loisirs}

La mise en controverse de l'ALAN résulte d'un processus amorcé par des astronomes professionnels à la fin des années 1950 aux États-Unis. On peut dater la première décision publique de protection de la qualité du ciel nocturne au 15 avril 1958, lorsque la ville de Flagstaff, en Arizona, adopte une réglementation de son éclairage public ${ }^{18}$ pour protéger l'activité des astronomes de l'observatoire Lowell (à partir duquel Pluton avait été découverte en 1930 par Clyde Tombaugh). En 1972, c'est

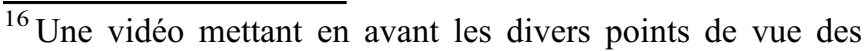
usagers a été réalisée par la commune dans le cadre de ce projet d'aménagement, www.youtube.com/watch? $\mathrm{v}=\mathrm{D}-\mathrm{rVzyovio}$.

${ }^{17}$ Comptes rendus des réunions publiques et résultats des enquêtes sont disponibles via le site de la ville de Crolles : www.ville-crolles.fr/vues/pages/la-mairie/grands-projets-eclai rage-public.php.

${ }_{18}$ «At its April 15, 1958 Council Meeting, the Mayor and Common Council of Flagstaff adopted Ordinance No. 440, an ordinance prohibiting the use of "any incandescent and arctype search light, beacon light or similar lighting device designed to and capable of projecting a beam of light into the sky for a distance of one half (1/2) mile". », http://flagstaff.az. gov/DocumentCenter/Home/View/15881.
}

la ville de Tucson qui emprunte le chemin tracé par Flagstaff suite à la mobilisation des astronomes de l'observatoire national de Kitt Peak qui dénoncent la dégradation de l'accessibilité au ciel étoilé (Challéat et Lapostolle, 2014). Suite au développement massif de l'automobilité, de l'étalement urbain et des faibles coûts de production de l'électricité qui engendrent l'installation d'un éclairage public toujours plus puissant, augmentant la taille et l'intensité des halos lumineux au-dessus des zones urbanisées, la problématique se diffuse en Europe dans les années 1970. On trouve les premières publications scientifiques traitant de ce problème dans des revues académiques d'astronomie. En France, l'astronome Jean Kovalevsky questionne le développement de l'éclairage urbain dès 1977 et fait adopter une résolution de l'Union astronomique internationale pour la protection des sites astronomiques (Kovalevsky, 1977). Avec son collègue François Barlier, il publie pour l'Académie des sciences un Rapport $d u$ groupe de travail sur la protection des observatoires astronomiques et géophysiques (Kovalevsky et Barlier, 1984) et diffuse la problématique auprès des astronomes professionnels (Kovalevsky, 1986). Une structure associative internationale - l'International Dark-Sky Association (IDA) - est créée en 1988 sous l'impulsion de David Crawford, astronome à l'observatoire de Kitt Peak, afin de porter problème et revendications au-delà du microcosme de l'astronomie professionnelle: auprès des astronomes amateurs, mais également des gestionnaires d'aires protégées ${ }^{19}$ et des décideurs politiques. Les bases de la controverse sociotechnique entourant l'éclairage urbain sont posées. Celle-ci trouve aujourd'hui encore de nombreux développements et ses contours, comme son contenu, restent mouvants (Challéat et Lapostolle, 2014).

\section{L'ALAN contre l'obscurité : les perturbations des fonctions écologiques de la nuit}

Les effets écologiques de l'ALAN sont une préoccupation de longue date pour les sciences du vivant et l'on en trouve trace dans les revues dès les années 1920 (Fox, 1925; Rowan, 1925; Lewis, 1927). Mais c'est au biologiste néerlandais Frans Johan

\footnotetext{
${ }^{19}$ Dès 1993, le National Park Service (NPS) reconnaît ainsi le ciel nocturne comme ressource à protéger et à surveiller : «Non-biological natural resources, such as geological, paleontological and Quaternary resources are important natural resources of the parks, as are air quality, night sky and natural quiet. ", www.nps.gov/cany/learn/nature/upload/Night Sky2003.pdf. La Natural Sounds and Night Skies Division sera par la suite créée au sein du NPS, www.nps.gov/orgs/1050/ index.htm; elle abrite la NPS National Night Sky Monitoring Team.
} 
Verheijen (1956) que l'on doit les premières études systématiques des effets et des impacts de l'ALAN sur plusieurs espèces animales. Il démontre notamment que la lumière artificielle peut constituer un piège pour de nombreux insectes, poissons et oiseaux (Verheijen, 1960). Durant les années 1970 et 1980, il confirme ces premiers résultats (Verheijen, 1981) et propose, en 1985, de nommer photopollution ces effets et impacts négatifs de l'éclairage artificiel nocturne sur la faune (Verheijen, 1985). Si nommer un problème ne suffit pas à le constituer en champ de recherche, cela y contribue : les années 1980 et 1990 seront les décennies durant lesquelles cette thématique s'affirmera - sous le terme plus générique de light pollution - dans les sciences du vivant.

Il est aujourd'hui établi que l'ALAN modifie profondément l'alternance naturelle jour/nuit en érodant l'obscurité dans l'espace (mitage, fractionnement) comme dans le temps (raccourcissement, voire disparition des périodes d'obscurité), mais également dans différentes longueurs d'onde. Une grande variété d'effets et d'impacts de l'ALAN sont aujourd'hui identifiés et documentés. Ils dérivent de perturbations dues à un environnement altéré par le niveau de lumière artificielle ou encore de mécanismes d'attraction, fixation ou répulsion dans lesquels les sources lumineuses elles-mêmes sont directement en cause. En aval, ce sont les comportements de prédation, de reproduction, de migration et de communication au sein de nombreuses populations qui s'en trouvent perturbés. L'évolution de ces comportements influence les communautés par l'intermédiaire des interactions entre espèces, notamment la compétition et la prédation. Par suite d'interactions inter et intraspécifiques, la structure d'une communauté peut être modifiée. Des impacts sont ainsi relevés sur des espèces n'étant pas directement soumises aux effets de la lumière artificielle, comme la perte d'une ressource pour un prédateur spécialisé ou la disparition en un lieu d'insectes pollinisateurs menaçant la diversité floristique. On trouvera dans l'ouvrage de référence de Rich et Longcore (2006), Ecological consequences of artificial night lighting, le détail de ces différents travaux, et chez Gaston et al., 2012, 2013, 2015 , une revue plus récente des recherches en la matière. L'ensemble de ces recherches amène Hölker et al., (2010) à ériger la pollution lumineuse en «menace globale pour la biodiversité ».

\section{L'ALAN contre les rythmes naturels: un facteur de risques sanitaires environnementaux}

La «théorie de la lumière artificielle nocturne» (light-at-night theory) voit le jour en 1987 sous la plume de Richard Stevens, épidémiologiste à l'Université du Connecticut. Dans un article intitulé «Electric power use and breast cancer: a hypothesis ", paru dans l'American Journal of Epidemiology, il émet l'hypothèse que
l'ALAN pourrait favoriser le développement du cancer du sein (Stevens, 1987). Partant, il entend expliquer en partie la croissance de ce risque à l'échelle internationale ainsi que certaines variations dans sa distribution spatiale. Les processus physiologiques justifiant, dans cette publication, les relations causales entre l'ALAN et cancer du sein n'ont pas tous été validés par les études plus approfondies qui ont suivi. Mais cette étude a néanmoins permis de mettre en lumière le rôle majeur de l'alternance lumière/obscurité dans la rythmicité circadienne de sécrétion de la mélatonine. Incidemment, elle a contribué à fonder un champ de recherche novateur et particulièrement fertile depuis le début des années 2000 : celui des impacts sanitaires de l'ALAN.

Il est aujourd'hui établi que l'ALAN affecte la santé humaine par le dérèglement du rythme nycthéméral ${ }^{20}$, de l'alternance naturelle d'un jour et d'une nuit, alternance correspondant à un cycle biologique de 24 heures. Pour l'être humain comme pour la plupart des espèces complexes, cette rythmicité jour/nuit est liée aux phases de veille et de sommeil, et l'alternance naturelle lumière/ obscurité est le premier «donneur de temps» pour l'horloge interne de l'homme. Une exposition à la lumière durant la nuit occasionne des troubles du nycthémère et peut participer d'un dérèglement de la sécrétion de la mélatonine (hormone chronobiotique dont la sécrétion déclenche à sa suite de nombreux processus tels que la régulation des cycles veille/sommeil, de la sécrétion du cortisol, de la température corporelle, du système immunitaire, de la pression sanguine, ou encore des métabolismes cellulaire et osseux). Parallèlement, les chercheurs montrent des effets non négligeables de la mélatonine sur la vitesse d'apparition et de développement de certains cancers. Les publications sur ces différentes questions sont nombreuses. On trouve chez Schernhammer et Schulmeister (2004), Megdal et al. (2005), Reiter et al. (2007), Navara et Nelson (2007) et dans les travaux récents de Stevens $(2009 ; 2011)$ des synthèses et des évaluations détaillées des recherches actuelles en la matière.

\section{L'environnement nocturne: l'avènement d'une notion socioécosystémique}

\section{L'institutionnalisation de l'environnement nocturne: des nuisances lumineuses aux paysages nocturnes}

La mise en évidence des coûts écologiques et sanitaires de l'ALAN enrichit l'«éclairer juste» des

\footnotetext{
${ }^{20}$ Rythme fonctionnel pour les espèces suivant la variation de luminosité du jour et de la nuit. Le rythme nycthéméral se différencie du rythme circadien par le fait qu'il est géré par l'intensité de la lumière.
} 
enjeux de la protection de l'environnement nocturne. En 2007 en France, l'Association nationale pour la protection du ciel et de l'environnement nocturne (ANPCEN) arrive, par l'intermédiaire de l'association France nature environnement (FNE), à porter la pollution lumineuse jusqu'à la table des négociations du Grenelle de l'environnement. L'article 36 du projet de loi Grenelle I de 2008 dispose : « Les émissions de lumière artificielle de nature à présenter des dangers ou à causer un trouble excessif aux personnes, à la faune, à la flore ou aux écosystèmes, entraînant un gaspillage énergétique ou empêchant l'observation du ciel nocturne feront l'objet de mesures de prévention, de suppression ou de limitation ». Le 29 juin 2010, la Chambre basse adopte le projet de loi portant engagement national pour l'environnement, loi dite «Grenelle II»: l'article 173 inscrit la prévention de la pollution lumineuse dans le Code de l'environnement. Le décret du 12 juillet $2011^{21}$ crée dans la partie réglementaire un chapitre spécifique à la pollution lumineuse, qui définit les installations concernées et le zonage permettant d'adapter les exigences aux enjeux spécifiques des espaces (agglomération, espaces naturels, sites astronomiques).

Le 17 août 2015, le problème de la pollution lumineuse est inscrit dans la loi relative à la transition énergétique pour la croissance verte ${ }^{22}$. Son traitement est adossé au plan climat-air-énergie territorial (PCAet) que les territoires - par l'intermédiaire des établissements publics de coopération intercommunale à fiscalité propre, ou par celui de l'établissement public chargé du schéma de cohérence territoriale (Scot) pour les territoires regroupés au sein d'un Scot-doivent mettre en œuvre. Là encore, c'est le Code de l'environnement qui se trouve modifié. Son article L229-26 ${ }^{23}$ dispose désormais que «Lorsque cet établissement public exerce la compétence en matière d'éclairage $[. .$.$] ce programme d'actions [le$ PCAET] comporte un volet spécifique à la maîtrise de la consommation énergétique de l'éclairage public et de ses nuisances lumineuses. »)

Le 8 août 2016, une troisième grande étape de reconnaissance institutionnelle de l'environnement nocturne est franchie. La loi pour la reconquête de la biodiversité,

\footnotetext{
$\overline{{ }^{21} \text { Décret } n^{\circ} 2011-831 \text { du }} 12$ juillet 2011 relatif à la prévention et à la limitation des nuisances lumineuses, Journal officiel, 13 juillet 2011, 0161, www.legifrance.gouv.fr/affichTexte.do? cidTexte $=$ JORFTEXT000024357936\&dateTexte $=\&$ categorie Lien $=\mathrm{id}$.

${ }^{22}$ Loi $\mathrm{n}^{\mathrm{o}} 2015-992$ du 17 août 2015 relative à la transition énergétique pour la croissance verte, Journal officiel, 18 août 2015, 0189, www.legifrance.gouv.fr/affichTexte.do?cidTexte= JORFTEXT000031044385\&categorieLien $=\mathrm{id}$.

${ }^{23}$ Article L229-26 du Code de l'environnement, www. legifrance.gouv.fr/affichCodeArticle.do?idArticle=LEGIAR TI000022476854\&cidTexte=LEGITEXT000006074220.
}

de la nature et des paysages ${ }^{24}$ entérine la dimension environnementale de la problématique de la pollution lumineuse en inscrivant explicitement la notion d'environnement nocturne dans le Code de l'environnement. L'article L110-2 dudit Code dispose ainsi désormais qu' " Il est du devoir de chacun de veiller à la sauvegarde et de contribuer à la protection de l'environnement, y compris nocturne. » Par ailleurs, une notion nouvelle fait son apparition, revendiquée de longue date par les astronomes $^{25}$ : les «paysages nocturnes ${ }^{26} »$. L'article L110-1 du Code de l'environnement est désormais ainsi formulé : «Les espaces, ressources et milieux naturels terrestres et marins, les sites, les paysages diurnes et nocturnes, la qualité de l'air, les êtres vivants et la biodiversité font partie du patrimoine commun de la nation. Ce patrimoine génère des services écosystémiques et des valeurs d'usage. »

Trois dispositifs légaux apparaissent donc en une dizaine d'années, qui établissent un nouveau principe directeur de l'aménagement en liant transition énergétique et protection de la biodiversité ${ }^{27}$. Cette liaison s'opérationnalise dans les dispositifs de territorialisation de l'environnement nocturne.

\footnotetext{
${ }^{24}$ Loi $\mathrm{n}^{\mathrm{o}} 2016-1087$ du 8 août 2016 pour la reconquête de la biodiversité, de la nature et des paysages, Journal officiel, 9 août, 0184, www.legifrance.gouv.fr/eli/loi/2016/8/8/ DEVL1400720L/jo\#JORFSCTA000033016238.

${ }^{25}$ Plus précisément, si la notion de « paysages nocturnes » n'est mobilisée que depuis peu par les associations de défense de l'environnement nocturne, le milieu de l'astronomie amateur opère de façon croissante, depuis les années 1980, un empaysagement de la nuit (Bénos et Challéat, 2014) qui peut être lu suivant le cadre d'analyse de l'extinction de l'expérience de nature (Miller, 2005 ; Fleury et Prévot, 2017). Voir, par exemple, le dernier paragraphe de «La pollution lumineuse du ciel nocturne» article publié en 1982 dans le $\mathrm{n}^{\mathrm{o}} 187$ de la revue Ciel et Espace, https://renoir.hypotheses. org/files/2017/10/Ciel-et-Espace-n\%C2\%B0-187-mai-juin1982-Ren\%C3\%A9-Verseau-La-pollution-lumineuse-du-cielnocturne.pdf.

${ }^{26} \mathrm{Il}$ est à noter que, suite aux évolutions numériques de la photographie (sensibilité des capteurs et montages en «timelapse» notamment), les paysages nocturnes magnifiés sont de plus en plus présents dans l'iconographie contemporaine de la wilderness et accompagnent les stratégies marketing de certains territoires (montagnes, déserts, territoires nordiques, etc.) (Challéat et al., 2015b).

${ }^{27}$ Un nouvel arrêté visant à réduire significativement la pollution lumineuse est par ailleurs en cours de préparation. Il prévoit notamment de rendre obligatoire pour les collectivités la mise en place d'un plan lumière comportant un volet visant à quantifier et à limiter l'impact de l'éclairage public sur la biodiversité.
} 


\section{La territorialisation de l'environnement nocturne : valorisation, protection, labellisation}

La territorialisation de l'environnement nocturne - que nous définissons comme l'«[...] établissement de zonages, de règles nouvelles, d'aires de protection qui entraînent une requalification de l'espace $[\ldots]^{28} \gg-$ se décline dans différentes modalités de l'action publique. Pour les unes, l'environnement nocturne est un objet d'activités récréatives prenant comme support la nuit «naturelle» et comme objets les phénomènes et les transformations de l'environnement qui lui sont liés (ciel étoilé, phénomènes célestes, bruits de la faune nocturne, etc.). Plusieurs territoires ruraux se saisissent actuellement de cet engouement pour l'environnement nocturne et l'érigent en ressource territoriale, notamment par des stratégies de développement (éco)touristique (Lapostolle et al., 2015). Pour les autres, il s'agit de diffuser cette préoccupation par des labels englobants qui établissent des règles nouvelles. C'est le cas du concours «Villes et villages étoilés $^{29} \gg$ qui fixe de nombreuses cibles :

« la promotion et la mise en œuvre, par les communes qui concourent, d'un éclairage extérieur visant à prévenir, limiter et supprimer les nuisances lumineuses et notamment soucieux des impacts sur la biodiversité et les paysages nocturnes, sur le sommeil et la santé des habitants, des économies d'énergies, de la limitation des gaz à effet de serre induits, de l'éco-conception et du recyclage des matériels utilisés, ainsi que de la capacité d'observation du ciel nocturne pour les générations actuelles et à venir ${ }^{30} »$.

Avec 570 communes ainsi primées depuis 2009, ce label diffuse la problématique des pollutions lumineuses ainsi que de «bonnes pratiques » en matière d'éclairage. Sa portée reste cependant très limitée et son mode d'action tient plus de la dissémination spatiale tous azimuts des marqueurs territoriaux du label (panneaux aux entrées d'agglomérations) que d'une réelle cohérence en termes de protection territorialisée de l'environnement nocturne.

Bien plus fortement, la territorialisation de l'environnement nocturne se lit dans une dernière modalité d'action : la mise en protection du ciel étoilé grâce à de nouveaux zonages construits sur la logique classique

\footnotetext{
${ }^{28}$ Raphaël Mathevet (2012, p. 35) définit ainsi la «territorialisation de la biodiversité». Nous reprenons à notre compte et en partie sa définition, et l'appliquons à l'environnement nocturne.

${ }^{29}$ Concours «labellisant», organisé par l'ANPCEN en partenariat avec le ministère de l'Écologie, les parcs nationaux de France, la Fédération des parcs naturels régionaux de France et la Ligue de protection des oiseaux.

${ }^{30}$ Extrait du règlement à consulter sur le site de l'ANPCEN, www.anpcen.fr/docs/20170113115622_14r3p2_doc198.pdf.
}

centre-périphérie. La dynamique de développement de « réserves de ciel étoilé » (Charlier et Bourgeois, 2013; Bénos et al., 2016) dans les espaces nord-américain et européen constitue le marqueur le plus prégnant de ce processus. Initié en 1993 aux États-Unis avec la création de la réserve de ciel étoilé du lac Hudson (Michigan), ce n'est qu'à la fin des années 2000 qu'il prend véritablement son essor. Il se base sur une logique de labellisation de qualité environnementale territoriale portée par différentes associations issues du Dark-sky movement, comme la Royal Astronomical Society of Canada (RASC) et l'IDA. Plus de 100 territoires sont, à l'heure actuelle et à l'échelle mondiale, labellisés par l'IDA suivant l'intensité des mesures de protection prises par les collectivités locales ${ }^{31}$. Ces zonages de protection du ciel étoilé ont initialement permis la valorisation de hauts lieux de l'observation astronomique et sont aujourd'hui recherchés par les aires protégées «classiques » qui élargissent ainsi la portée de leurs mesures de protection au ciel étoilé.

En décembre 2013, la réserve internationale de ciel étoilé (RICE) du pic du Midi est labellisée par l'IDA. Ce label reconnaît à la fois la qualité exceptionnelle du ciel étoilé haut-pyrénéen et le projet de territoire pour le préserver et le valoriser. Le référentiel de la transition énergétique a joué un rôle prépondérant dans l'appropriation de cette démarche par les élus locaux, en sus de la dimension touristique fortement présente (Bénos et al., 2016). Mais au-delà, la RICE du pic du Midi adjoint aujourd'hui à la protection du ciel étoilé la protection de la biodiversité. Elle tente, avec l'aide du parc national des Pyrénées, de territorialiser une "trame noire ${ }^{32}$ » (Challéat, 2010; Sordello, 2017b). Il s'agit ici de définir et de restaurer les réservoirs de biodiversité et les continuités écologiques nocturnes afin d'endiguer la fragmentation des habitats (Jongman, 2002) et l'imperméabilisation écologique des espaces par l'ALAN (Sordello, 2017a). En appui sur le cadre de travail du réseau écologique (Boitani et al., 2007), la logique réticulaire de la trame noire vise donc une plus grande intégration des dynamiques temporelles des processus écologiques dans la planification

\footnotetext{
${ }^{31}$ L'IDA décerne les labels suivants, dans l'ordre décroissant d'intensité des mesures de protection exigées pour leur obtention: International dark sky sanctuaries, International dark sky reserves, International dark sky parks, International dark sky communities, Urban night sky places et Dark sky developments of distinction, http://darksky.org/idsp/.

${ }^{32}$ Un débat sémantique existe autour de la dénomination «trame noire», et d'autres termes comme «trame sombre» (terme utilisé par le parc national des Pyrénées) ou encore «trame étoilée» (terme promotionnel utilisé par l'ANPCEN dans le cadre du concours «Villes et villages étoilés ») existent actuellement pour désigner peu ou prou cette notion. C'est par souci de cohérence avec le code couleurs de la TVB dont elle s'inspire que nous utilisons «trame noire» comme unique dénomination.
} 

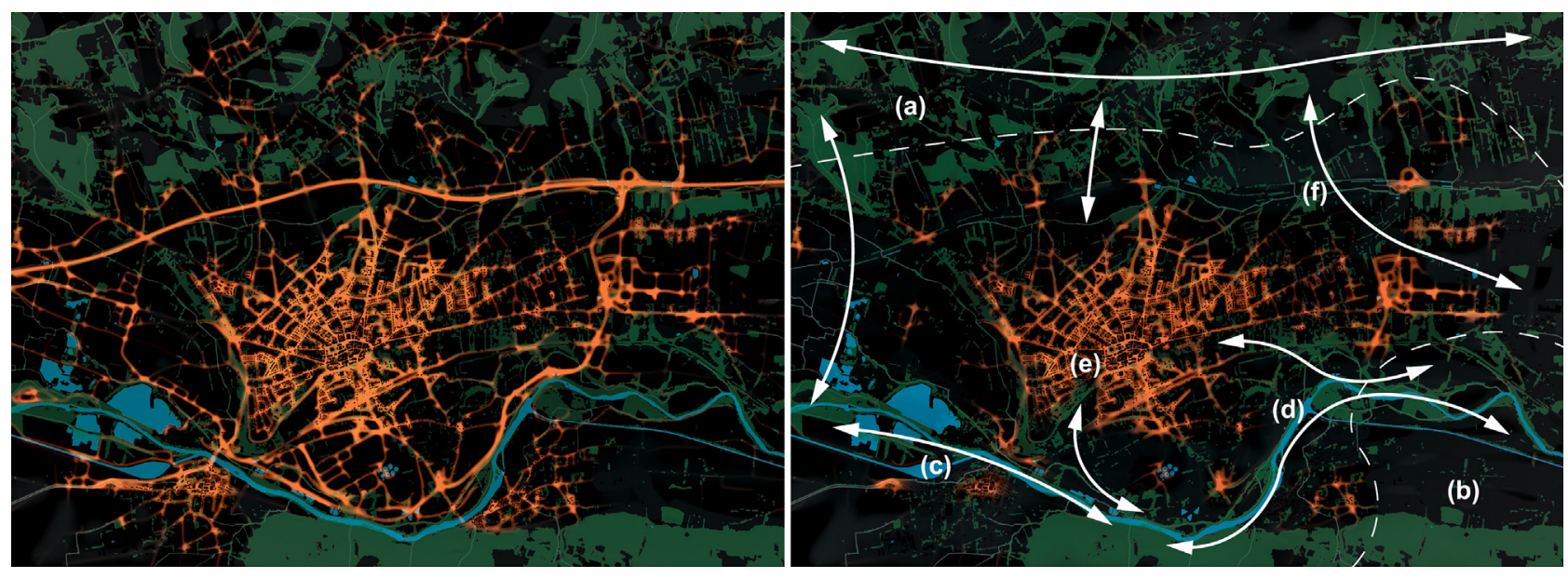

Fig. 1. La «trame noire», un outil d'aménagement pour lutter contre la fragmentation des habitats par la lumière artificielle (réalisation: S. Challéat).

Simulation d'une politique de réduction globale des intensités de l'ALAN (diminution du halo lumineux) et, localement, de restauration des continuités écologiques nocturnes. Une «trame noire» permet de lutter contre la fragmentation des habitats par l'ALAN via une réduction du mitage des espaces (a), l'identification et la protection de réservoirs de biodiversité nocturne (b), la restauration de continuités écologiques (f) et de corridors noirs $(c+d)$, ou encore l'augmentation de la perméabilité écologique entre espaces urbains et ruraux (e).

de la conservation de la biodiversité, ainsi qu'un approfondissement et une extension de la lutte contre la pollution lumineuse en direction de la biodiversité ordinaire et au sein des espaces ordinaires, ruraux comme urbains (Fig. 1).

La ville de Lille travaille ainsi à la restauration des continuités écologiques nocturnes et souhaite expérimenter une trame noire dans le parc de la Citadelle et le long du Canal de la Deûle ${ }^{33}$. De son côté, la ville de Rennes a inscrit la trame noire dans le cahier des charges de son nouveau schéma directeur d'aménagement lumière $\left(\mathrm{SDAL}^{34}\right)$ : certains espaces sont volontairement laissés dans l'obscurité. La trame marque ainsi une rupture avec la protection zonée classique, souvent réservée aux espaces abritant une «nature remarquable»: la logique réticulaire permet la diffusion spatiale de la protection de la biodiversité. Les évolutions de la lutte contre la pollution lumineuse illustrent particulièrement bien ces changements : en liant biodiversité et transition énergétique, la territorialisation de l'environnement nocturne donne à voir de nouveaux principes d'aménagement de l'espace.

\footnotetext{
${ }^{33}$ Voir www.adu-lille-metropole.org/wp-content/uploads/ 2017/09/cahier7.pdf.

${ }^{34}$ Le SDAL de Rennes est réalisé par l'agence Concepto: «Dans cette étude, la forme urbaine de Rennes a été un terrain particulièrement propice à l'élaboration de principes permettant d'apporter de l'obscurité en ville et de moduler l'éclairage selon les besoins et les pratiques nocturnes. La pierre angulaire du schéma directeur d'aménagement lumière a donc été la réalisation d'une trame noire, venant compléter les trames verte et bleue rennaises.», www.concepto.fr/portfolio_page/ schema-directeur-damenagement-lumiere-rennes-france/.
}

\section{L'environnement nocturne, champ fertile pour l'approche socioécosystémique}

L'environnement nocturne est aujourd'hui transcrit dans des projets de territoire. Ce processus participe d'un «transfert de l'écologie à l'aménagement du territoire, qui s'accompagne d'une interprétation qui met en valeur de nouvelles priorités » (Franchomme et al., 2013). La réduction de la pression lumineuse sur l'environnement pour protéger la biodiversité nocturne et préserver les services écosystémiques (qu'ils soient culturels, de régulation ou de support [Gallaway, 2015; Lyytimäki, 2013]) liés à l'obscurité est une de ces nouvelles priorités. Cette déclinaison spatiale des problèmes environnementaux signe un profond changement de paradigme qui remodèle nos cadres de pensée et d'analyse des relations hommes/milieux. La complexité a permis de penser les dimensions systémiques et interactionnelles entre éconosphère et biosphère, sociosystèmes et écosystèmes. Mais plus encore, plusieurs travaux (Latour, 2015; Descola, 2005; Serres, 1990) participent de la déconstruction de ces divisions. Le cadre de travail interdisciplinaire que pose Ostrom (2009) autour des social-ecological systems traduit la volonté de mieux intégrer les dynamiques d'interaction entre système écologique et système social. Il permet de résoudre en grande partie le schisme actuellement existant - par exemple autour de la notion d'environnement - entre approches par les sciences de la société et approches par les sciences biophysiques (Janssen et al., 2011).

La construction et la légitimation scientifique, sociale et territoriale de la pollution lumineuse révèlent que 


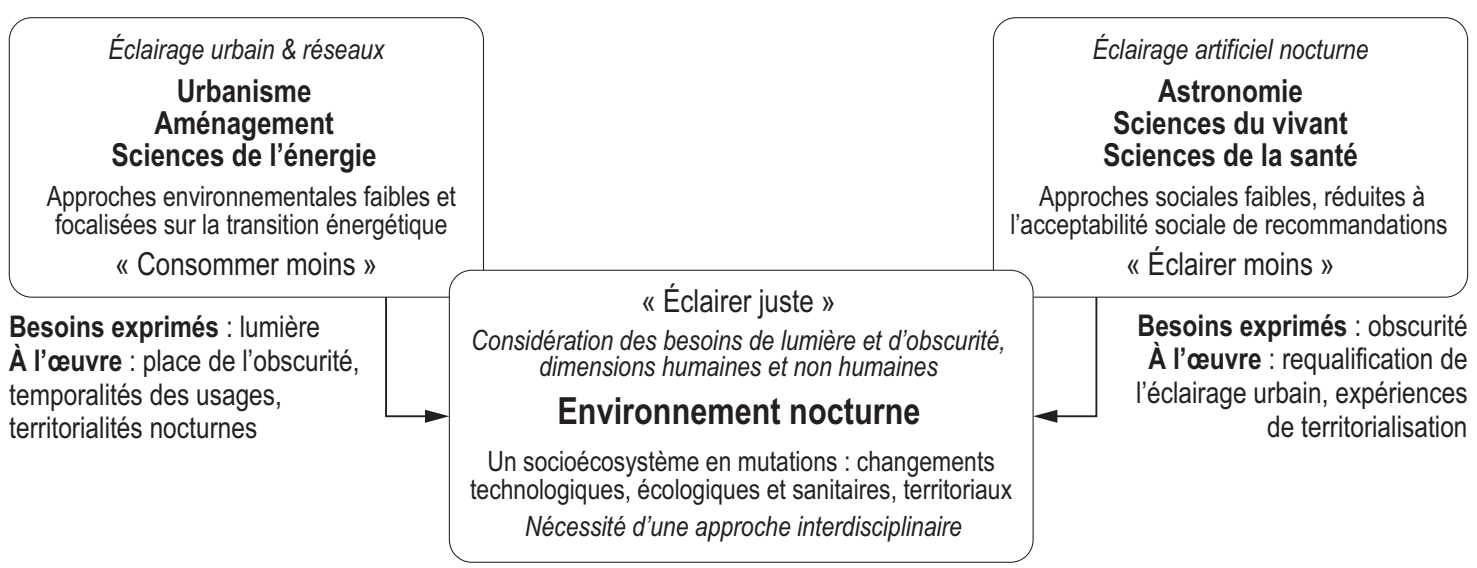

Fig. 2. L'environnement nocturne ou la construction d'un nouvel objet de recherche (réalisation: S. Challéat).

L'environnement nocturne résulte ainsi de la requalification environnementale de l'éclairage urbain suite aux travaux des astronomes, des écologues et des médecins, et trouve une transcription dans le référentiel d'action de «l'éclairer juste». Comme nouvel objet de recherche, il opère la jonction entre approches urbanistiques de l'éclairage et approches par les sciences du vivant.

l'ALAN n'est pas que bénéfices mais également objet de dommages, et de plus en plus fréquemment perçu comme tel. La notion d'environnement nocturne - qui entend dépasser le seul problème de la pollution lumineusenécessite de mieux connaître les perceptions et les usages de la lumière et de l'obscurité. Autrement dit, il s'agit de provoquer le débat sur ce qui justifie la protection de l'environnement nocturne et sur les modalités de sa mise en œuvre. Ces réflexions sont fortement liées à la connaissance produite par les sciences du vivant et de la santé quant aux dommages infligés à la «ressource obscurité ». Elles ne peuvent cependant pas être menées sur la seule base de grandeurs physiques traduites en indicateurs " extériorisés », hors-sol et hors individus et doivent donc composer avec les approches et les résultats des sciences de la société, car «à chaque fois qu'il y a délimitation, classification et quantification du vivant, une vision du monde s'exprime et des valeurs particulières sont mobilisées » (Devictor, 2015, p. 110).

\section{Conclusion}

Considéré quasi exclusivement en termes de bénéfices par les champs de l'urbanisme et de l'aménagement, l'éclairage artificiel nocturne est aujourd'hui requalifié en nuisance, voire en pollution ${ }^{35}$. Cette requalification

\footnotetext{
${ }^{35}$ Concernant la distinction théorique que nous établissons entre nuisance lumineuse et pollution lumineuse, voir Challéat, 2011 (p. 190-191). Mais au-delà des définitions et des travaux scientifiques - qui aujourd'hui valident l'usage du terme pollution lumineuse - cette distinction recouvre des enjeux politiques: le choix des termes n'est pas neutre et détermine l'étendue de la controverse sociotechnique entourant l'ALAN. Voir à ce propos Challéat et Lapostolle, 2014 (p. 318).
}

environnementale fait suite à l'émergence des savoirs développés par les sciences expérimentales, et plus particulièrement les sciences du vivant et de la santé. La mise en évidence des coûts écologiques et sanitaires de cet outil d'aménagement enrichit l' «éclairer juste» des enjeux de la protection de l'environnement nocturne (Fig. 2). Les différentes reconnaissances législatives des enjeux de la pollution lumineuse légitiment de nouvelles expériences de territorialisation de l'environnement nocturne, entre processus de protection, de valorisation et de labellisation. De nouveaux outils à destination des décideurs territoriaux et plus particulièrement des praticiens de la conservation font leur apparition: la protection de l'environnement nocturne devient un nouveau principe d'aménagement du territoire. Suivant le modèle taches-corridors-matrice, les réserves internationales de ciel étoilé et autres zonages initialement fondés sur les besoins des astronomes peuvent être considérés comme les réservoirs de biodiversité nocturne d'une «macro trame noire». À une échelle plus fine, cette trame noire permet de diffuser la protection de l'environnement nocturne jusque dans les espaces ordinaires, urbains compris.

Ces transcriptions territoriales de la protection de l'environnement nocturne ne sont pour autant pas stabilisées et il convient certes de les observer, mais également de les alimenter de façon réflexive afin qu'elles ne soient pas construites hors-sol et hors individus usagers des espaces-temps nocturnes. Les dimensions sociales et environnementales sont trop souvent disjointes dans les décisions individuelles comme dans les politiques publiques, et l'appréhension des impacts environnementaux sous forme de grandeurs physiques et non d'effets sur les individus accentue cette dissociation. La démocratie territoriale est donc l'un des enjeux majeurs de la protection de l'environnement nocturne comme nouveau 
principe directeur de l'aménagement. Comme le soulignent Mathevet et al., (2012), «[...] les décisions doivent être prises au niveau d'organisation où celles-ci génèrent leurs conséquences. Ceci implique la nécessité d'informer les acteurs et de les responsabiliser en les associant à la gestion du patrimoine naturel [...]». En ce sens, l'objet de recherche «environnement nocturne » a grand besoin d'être étudié dans une approche réellement interdisciplinaire et pluriactorielle, suivant le cadre d'analyse des socioécosystèmes.

\section{Remerciements}

Je tiens à remercier l'ensemble des membres du collectif Renoir pour les discussions et les échanges ayant permis d'alimenter, depuis 2013, les réflexions exposées dans cet article: Clémentine Azam, Rémi Bénos, Nicolas Bourgeois, Pierre-Olivier Dupuy, Frédérique Girard, Dany Lapostolle, Johan Milian et Thomas Poméon. Je remercie mes collègues Christelle Hinnewinckel et Magalie Franchomme (Université Lille 1, EA4477 TVES) de m'avoir permis une observation participante au sein du programme Tramenoire (appel à projets de recherche Biodiversité 2014, région Nord-Pas-de-Calais). Je remercie également les structures territoriales m'ayant associé à leurs démarches de territorialisation de l'environnement nocturne: la RICE du pic du Midi, le parc national des Pyrénées, le parc national des Cévennes, le parc naturel régional des Pyrénées ariégeoises et le parc naturel régional des Baronnies provençales. Enfin, je remercie l'ensemble des acteurs socioprofessionnels rencontrés au fil des entretiens et des déplacements sur mes terrains de recherche depuis 2006.

\section{Références}

Beauparlant C., Darris G., Lemoine A., Léon H., 2006. La ville, la nuit. Rennes et Nantes, de nouvelles exigences de gestion urbaine, Les Cahiers de la sécurité, 61, 2, 85-107, www.urbansecurity.be/IMG/pdf/groupe_reflex_La_ville_ la_nuit.pdf.

Bénos R., Challéat S., 2014. «Faire advenir le noir»: production de paysages nocturnes et contemporanéité de la faible densité, Carnets du collectif Renoir -Ressources environnementales nocturnes, tourisme, territoires, http:// renoir.hypotheses.org/593.

Bénos R., Challéat S., Lapostolle D., Dupuy P.-O., Poméon T., Milian J., Girard F., 2016. La protection de la nuit d'un haut lieu touristique de montagne: la réserve internationale de ciel étoilé du Pic du Midi comme nouvelle ressource territoriale, in Delaplace M., Gravari-Barbas M. (Eds), Nouveaux territoires touristiques: invention, reconfigurations, repositionnements, Québec, Presses de l'Université du Québec, 55-82.
Bertin S., Paquette S., 2015. Apprendre à regarder la ville dans l'obscurité : les «entre-deux» du paysage urbain nocturne, Environnement Urbain/Urban Environment, 9, http://eue. revues.org/603.

Boitani L., Falcucci A., Maiorano L., Rondinini C., 2007. Ecological networks as conceptual frameworks or operational tools in conservation, Conservation Biology, 21, 6, 1414-1422, doi: 10.1111/j.1523-1739.2007.00828.x.

Bourgeois N., 2016.La protection du ciel étoilé: approche de la construction sociale et de la mise en ouvre d'une pratique émergente. Thèse de doctorat en géographie, Pau, Université de Pau et des pays de l'Adour.

Brands J., Schwanen T., van Aalst I., 2015. Fear of crime and affective ambiguities in the night-time economy, Urban Studies, 52, 3, 439-455, doi: 10.1177/0042098013505652.

Challéat S., 2010. "Sauver la nuit». Empreinte lumineuse, urbanisme et gouvernance des territoires. Thèse de doctorat en géographie, Dijon, Université de Bourgogne.

Challéat S., 2011. La nuit, une nouvelle question pour la géographie, Bulletin de l'Association de Géographes Français, 88, 2, 183-196, www.persee.fr/doc/ bagf_0004-5322_2011_num_88_2_8217.

Challéat S., 2016. La noche, nuevo objeto de salvaguarda, in Monod Becquelin, A., Galinier J. (Eds), Las cosas de la noche. Una mirada diferente, México, Centro de estudios mexicanos y centroamericanos, http://books.openedition. org/cemca/4201.

Challéat S., Lapostolle D., 2014. (Ré)concilier éclairage urbain et environnement nocturne: les enjeux d'une controverse sociotechnique, Natures Sciences Sociétés, 22, 4, 317-328, doi: $10.1051 / \mathrm{nss} / 2014045$.

Challéat S., Lapostolle D., 2017. Prendre en compte les usages pour mieux éclairer la nuit, Métropolitiques, www. metropolitiques.eu/Prendre-en-compte-les-usages-pourmieux-eclairer-la-nuit.html.

Challéat S., Lapostolle D., Bénos R., 2015a. Consider the darkness. From an environmental and sociotechnical controversy to innovation in urban lighting, ArticuloJournal of Urban Research, 11, https://articulo.revues.org/ 3064.

Challéat S., Dupuy P.-O., Bénos R., Girard F., Poméon T., Lapostolle D., Milian J., 2015b. The role of the Northern lights in the production of a new touristic imaginary of the Nordic, in The dynamics of darkness in the North. Conference abstracts booklet, 10th International Multidisciplinary Conference of the International Laboratory for the Comparative Multidisciplinary Study of Representations of the North, February 26th to 28th, Reykjavík, Iceland, Montréal, Université du Québec à Montréal, www. imaginairedunord.uqam.ca/pdf/150209\%20Livret\% 20modifie.pdf.

Charlier B., Bourgeois N., 2013. « Half the park is after dark». Les parcs et réserves de ciel étoilé: nouveaux concepts et outils de patrimonialisation de la nature, L'Espace géographique, 42, 3, 200-212, doi: 10.3917/eg.423.0200.

Comelli C., 2015. Mutations urbaines et géographie de la nuit à Bordeaux. Thèse de doctorat en géographie, Bordeaux, 
Université Bordeaux Montaigne, https://core.ac.uk/down load/pdf/46812417.pdf.

Deleuil J.-M., 2009. Éclairer la ville autrement. Innovations et expérimentations en éclairage public, Lausanne, Presses polytechniques et universitaires romandes.

Deleuil J.-M., Toussaint J.-Y., 2000. De la sécurité à la publicité, l'art d'éclairer la ville, Les Annales de la Recherche Urbaine, 87, 52-58, www.annalesdelarecher cheurbaine.fr/IMG/pdf/Deleuil.Toussaint_ARU_87.pdf.

Descola P., 2005. Par-delà nature et culture, Paris, Gallimard.

Devictor V., 2015. Nature en crise. Penser la biodiversité, Paris, Seuil.

Edensor T., 2015. The gloomy city: rethinking the relationship between light and dark, Urban Studies, 52, 3, 422-438, doi: 10.1177/0042098013504009.

Fleury C., Prévot A.-C., 2017. Le souci de la nature. Apprendre, inventer, gouverner, Paris, CNRS Éditions.

Fouquet R., Pearson P.J.G., 2006. Seven centuries of energy services: the price and use of light in the United Kingdom (1300-2000), The Energy Journal, 27, 1, 139-177, www. jstor.org/stable/23296980.

Fox H.M., 1925. The effect of light on the vertical movement of aquatic organisms, Biological Reviews, 1, 4, 219-224, doi: 10.1111/j.1469-185X.1925.tb00550.x.

Franchomme M., Bonnin M., Hinnewinkel C., 2013. La biodiversité «aménage-t-elle» les territoires? Vers une écologisation des territoires, Développement durable \& territoires, 4, 1, http://developpementdurable.revues.org/9749.

Gallaway T., 2015. The value of night sky, in Meier J., Hasenöhrl U., Krause K., Pottharst M. (Eds), Urban lighting, light pollution, and society, New York, Routledge, 267-283.

Gaston K.J., Davies T.W., Bennie J., Hopkins J., 2012. Reducing the ecological consequences of night-time light pollution: options and developments, Journal of Applied Ecology, 49, 6, 1256-1266, doi: 10.1111/ j.1365-2664.2012.02212.x.

Gaston K.J., Bennie J., Davies T.W., Hopkins J., 2013. The ecological impacts of nighttime light pollution: a mechanistic appraisal, Biological reviews, 88, 4, 912-927, doi: 10.1111/brv.12036.

Gaston K.J., Gaston S., Bennie J., Hopkins J., 2015. Benefits and costs of artificial nighttime lighting of the environment, Environmental Reviews, 23, 1, 14-23, doi: 10.1139/er2014-0041.

Gwiazdzinski L. (Ed.), 2003. La ville 24heures sur 24. Regards croisés sur la société en continu, La Tour d'Aigues/Paris, L'Aube/Datar.

Gwiazdzinski L., 2015. «Introduction». The urban night: a space time for innovation and sustainable development, Articulo-Journal of Urban Research, 11, doi: 10.4000/ articulo. 3140.

Hadfield P., Lister S., Traynor P., 2009. 'This town's a different town today'. Policing and regulating the night-time economy, Criminology \& Criminal Justice, 9, 4, 465485, doi: $10.1177 / 1748895809343409$.

Hadfield P., 2014. The night-time city. Four modes of exclusion: reflections on the Urban Studies special collection, Urban Studies, 52, 3, 606-616, doi: 10.1177/ 0042098014552934.

Hobbs D., 2003. Bouncers: violence and governance in the night-time economy, Oxford/New York, Oxford University Press.

Hölker F., Wolter C., Perkin E.K., Tockner K., 2010. Light pollution as a biodiversity threat, Trends in Ecology \& Evolution, 25, 12, 681-682, doi: 10.1016/j.tree.2010.09.007.

Hollands R., 2002. Divisions in the dark: youth cultures, transitions and segmented consumption spaces in the nighttime economy, Journal of Youth Studies, 5, 2, 153-171, http://dx.doi.org/10.1080/13676260220134421.

Janssen M.A., Bousquet F., Ostrom E., 2011. A multimethod approach to study the governance of social-ecological systems, Natures Sciences Sociétés, 19, 4, 382-394, doi: $10.1051 / \mathrm{nss} / 2011135$.

Jongman R.H.G., 2002. Homogenisation and fragmentation of the European landscape: ecological consequences and solutions, Landscape and Urban Planning, 58, 2, 211-221, doi: 10.1016/S0169-2046(01)00222-5.

Kostic M., Djokic L., 2009. Recommendations for energy efficient and visually acceptable street lighting, Energy, 34, 10, 1565-1572, doi: 10.1016/j.energy.2009.06.056.

Kovalevsky J., 1977. La XVI ${ }^{\mathrm{e}}$ assemblée générale de l'Union astronomique internationale à Grenoble, L'Astronomie, 91, 99-115, http://articles.adsabs.harvard.edu//full/ 1977LAstr..91...99K/0000099.000.html.

Kovalevsky J., 1986. La protection des sites astronomiques, L'Astronomie, 100, 219-226, http://articles.adsabs.harvard. edu//full/1986LAstr.100..219K/0000219.000.html.

Kovalevsky J., Barlier F., 1984. Rapport du groupe de travail sur la protection des observatoires astronomiques et géophysiques, Paris, Gauthiers-Villars.

Lapostolle D., Challéat S., Milian J., Dupuy P.-O., 2015. L'appropriation de la transition énergétique par la protection des ressources environnementales nocturnes: expériences dans les Pyrénées et les Alpes du sud, Géocarrefour, 90, 4, 351-360, http://journals.openedition.org/geocarrefour.9967.

Lapostolle D., Doidy E., Borel M., Gateau M., 2016. L'habitat durable sans l'habiter? Fabrique de la densité en Bourgogne, Sciences de la société, 98, 14-23.

Latour B., 2015. Face à Gaïa. Huit conférences sur le nouveau régime climatique, Paris, La Découverte.

Lewis H.F., 1927. Destruction of birds by lighthouses in the provinces of Ontario and Quebec, Canadian FieldNaturalist, 41, 55-58.

Lovatt A., O'Connor J., 1995. Cities and the night-time economy, Planning Practice and Research, 10, 2, 127-134, doi: 10.1080/02697459550036676.

Lyytimäki J., 2013. Nature's nocturnal services: light pollution as a non-recognised challenge for ecosystem services research and management, Ecosystem Services, 3, 44-48, doi: 10.1016/j.ecoser.2012.12.001.

Mallet S., 2009. Des plans-lumière nocturnes à la chronotopie. Vers un urbanisme temporel. Thèse de doctorat, Marne-laVallée, Université Paris-Est.

Mathevet R., 2012. La solidarité écologique. Ce lien qui nous oblige, Arles, Actes Sud. 
Mathevet R., Thompson J., Bonnin M., 2012. La solidarité écologique: prémices d'une pensée écologique pour le $\mathrm{XXI}^{\mathrm{E}}$ siècle?, Écologie \& politique, 44, 1, 127-138, https:// cairn.info/revue-ecologie-et-politique1-2012-1-page-127.htm.

Megdal S.P., Kroenke C.H., Laden F., Pukkala E., Schernhammer E.S., 2005. Night work and breast cancer risk: a systematic review and meta-analysis, European Journal of Cancer, 41, 13, 2023-2032, doi: $10.1016 / \mathrm{j}$. ejca.2005.05.010.

Meier J., Hasenöhrl U., Krause K., Pottharst M. (Eds.), 2015. Urban lighting, light pollution and society New York, Routledge.

Miller J.R., 2005. Biodiversity conservation and the extinction of experience, Trends in Ecology \& Evolution, 20, 8, 430434, doi: 10.1016/j.tree.2005.05.013.

Mosser S., Devars J.-P., 2000. Quel droit de cité pour l'éclairage urbain? Une composante de la qualité des villes la nuit, Les Annales de la Recherche Urbaine, 87, 1, 63-72, doi: 10.3406/aru.2000.2337.

Navara K.J., Nelson R.J., 2007. The dark side of light at night: physiological, epidemiological, and ecological consequences, Journal of Pineal Research, 43, 3, 215-224, doi: 10.1111/j.1600-079X.2007.00473.x.

Nye D.E., 2015. The transformation of American urban space. Early electric lighting, 1875-1915, in Meier J., Hasenöhrl U., Krause K., Pottharst M. (Eds.), Urban lighting, light pollution and society, New York, Routledge, 30-45.

Ostrom E., 2009. A general framework for analyzing sustainability of social-ecological systems, Science, 325, 5939, 419-422, doi: 10.1126/science.1172133.

Painter K., 1996. The influence of street lighting improvements on crime, fear and pedestrian street use, after dark, Landscape and Urban Planning, 35, 2-3, 193-201, doi: 10.1016/0169-2046(96)00311-8.

Pieroni R., 2014. La nuit en ville et les nuisances sonores, cet ennemi intime. La régulation par les dispositifs sociotechniques, Urbanités, www.revue-urbanites.fr/wp-content/ uploads/2014/08/Urbanite\%CC\%81s-Chroniques-Pieroni. pdf.

Radulovic D., Skok S., Kirincic V., 2011. Energy efficiency public lighting management in the cities, Energy, 36, 4, 1908-1915, doi: 10.1016/j.energy.2010.10.016.

Raffestin C., 1988. Le territoire, la territorialité et la nuit, Actualités psychiatriques, 2, 48-50 (version post-print disponible sur archive ouverte UNIGE, https://archiveouverte.unige.ch/unige:4358).

Reiter R.J., Tan D.-X., Korkmaz A., Erren T.C., Piekarski C., Tamura H., Manchester L.C., 2007. Light at night, chronodisruption, melatonin suppression, and cancer risk: a review, Critical Reviews in Oncogenesis, 13, 4, 303-328, doi: 10.1615/CritRevOncog.v13.i4.30.
Rich C., Longcore T., 2006. Ecological consequences of artificial night lighting, Washington (DC), Island Press.

Rowan W., 1925. Relation of light to bird migration and developmental changes, Nature, 115, 494-495, doi: 10.1038/115494b0.

Schernhammer E., Schulmeister K., 2004. Invited review: light at night and cancer risk, Photochemistry and Photobiology, 79, 4, 316-318, doi: 10.1111/j.1751-1097.2004.tb00014.x.

Serres M., 1990. Le contrat naturel, Paris, Bourin.

Sordello R., 2017a. Les conséquences de la lumière artificielle nocturne sur les déplacements de la faune et la fragmentation des habitats: une revue, Bulletin de la Société des naturalistes luxembourgeois, 119, 39-54, www.snl.1u/ publications/bulletin/SNL_2017_119_039_054.pdf.

Sordello R., 2017b. Trame verte et bleue: toutes ces autres trames dont il faudrait aussi se préoccuper, Société française d'écologie et d'évolution. Regards et débats sur la biodiversité, 72, www.sfecologie.org/regard/r72mai-2017-r-sordello-corridors-ecologiques/.

Stevens R.G., 1987. Electric power use and breast cancer: a hypothesis, American Journal of Epidemiology, 125, 4, 556-561, doi: 10.1093/oxfordjournals.aje.a114569.

Stevens R.G., 2009. Light-at-night, circadian disruption and breast cancer: assessment of existing evidence, International Journal of Epidemiology, 38, 4, 963-970, doi: 10.1093/ ije/dyp 178 .

Stevens R.G., 2011. Testing the light-at-night (LAN) theory for breast cancer causation, Chronobiology International, 28, 8, 653-656, doi: 10.3109/07420528.2011.606945.

Van Liempt I., van Aalst I., Schwanen T., 2015. Introduction: geographies of the urban night, Urban Studies, 52, 3, $407-$ 421, doi: 10.1177/0042098014552933.

Verheijen F.J., 1956. On a method for collecting and keeping clupeids for experimental purposes, together with some remarks on fishery with light-sources and a short description of free cupulae of the lateral line organ on the trunk of the sardine Clupea pilchardus, Pubblicazioni della Stazione zoologica di Napoli, 28, 225-240.

Verheijen F.J., 1960. The mechanisms of the trapping effect of artificial light sources upon animals, Archives Néerlandaises de Zoologie, 13, 1, 1-107, doi: 10.1163/ $036551660 X 00017$.

Verheijen F.J., 1981. Bird kills at tall lighted structures in the USA in the period 1935-1973 and kills at a Dutch lighthouse in the period 1924-1928 show similar lunar periodicity, Ardea, 69, 2, 199-203.

Verheijen F.J., 1985. Photopollution: artificial light optic spatial control systems fail to cope with. Incidents, causation, remedies, Experimental Biology, 44, 1, 1-18, www.ncbi.nlm.nih.gov/pubmed/3896840.

Citation de l'article : Challéat S., 2018. Le socioécosystème environnement nocturne: un objet de recherche interdisciplinaire. Nat. Sci. Soc. 26, 3, 257-269. 